\title{
Exploring vulnerabilities to sepsis in Canada
}

\author{
Niranjan Kissoon, MBBS, MCCM, FRCP(C), FAAP, FACPE $\cdot$ John Mark Ansermino, MBBCh, MMed (Anaesth), \\ MSc (Info), FRCPC FFA (SA)
}

Received: 2 October 2019/Revised: 2 October 2019/Accepted: 18 October 2019/Published online: 25 November 2019

(c) Canadian Anesthesiologists' Society 2019

Epidemiology and surveillance systems for sepsis are rudimentary, highlighting how little we know of this common devastating condition. ${ }^{1,2}$ In an era of escalating healthcare costs and limited financial resources, we need to make strategic decisions regarding allocation of public health dollars. This allocation should be informed by robust granular data and should consider the other competing priorities based on the local context. Nevertheless, data acquisition and interpretation to comprehend the epidemiology of sepsis has been hampered by the protean signs and symptoms of sepsis, reflected in shifting diagnostic criteria. These limitations have led to an elusive target in quantifying the burden of sepsis.

Allocation of resources for prevention and optimal acute care and follow-up of patients with sepsis relies on identification of vulnerable populations. Indeed, sociodemographic factors including inequities have historically contributed to the causes, distribution, and outcomes of infectious diseases and sepsis globally. ${ }^{3}$ Hospital records provide scant information of the socio-demographic factors that underpin vulnerability and may be associated with sepsis. This lack of data means that most studies reporting critical care and hospital outcomes are not very useful to guide resource use, decrease inequities in care, or target prevention strategies for sepsis.

N. Kissoon, MBBS, MCCM, FRCP(C), FAAP, FACPE ( $₫)$ Critical Care - Global Child Health, Department of Pediatrics and Emergency Medicine, University of British Columbia, 4480 Oak Street, Vancouver, BC V6H 3V4, Canada

e-mail: nkissoon@cw.bc.ca

J. M. Ansermino, MBBCh, MMed (Anaesth), MSc (Info), FRCPC FFA (SA)

Department of Anesthesiology, Pharmacology \& Therapeutics, University of British Columbia, Vancouver, BC, Canada
In an attempt to provide a comprehensive portrait of socio-demographic characteristics that may be associated with sepsis, Hennessy et $a l^{4}$ linked census data from Statistics Canada to Canadian Institute for Health Information (CIHI) Discharge Abstract Database (DAD). Canadian census data contain information pertaining to income, education, employment, ethnicity, visible minority and immigrant status as well as functional limitations, while the DAD contains demographic (albeit scant), administrative, and clinical data from across Canada (excluding Quebec). The DAD data of patients $18 \mathrm{yr}$ or older who were admitted between 2006/2007 and 2008/ 2009 were linked to 2006 census data. The authors found that the age-standardized rate of sepsis hospitalization was 96 cases per 100,000 population; and of those, 37 cases per 100,000 population were classified as severe sepsis. Linkages of the databases revealed that male sex, never being married, visible minority status, functional limitations, and not being in the labour force were all associated with increased odds of hospitalization for sepsis. These variables most likely reflect socio-demographic inequities and vulnerable populations.

While the findings of this report are not surprising, the strength of this study is the linking of socio-demographic indices that may be an indication of vulnerability to sepsis and hospitalization. That vulnerability to sepsis and inequities are linked is reflected by the stark difference in sepsis incidence and outcomes between and within countries. ${ }^{1,2,5}$ That inequity in socioeconomic resources and access to healthcare contribute to sepsis burden and outcomes is highlighted by reports from Australia ${ }^{6}$ and the United States. ${ }^{7}$ Moreover, the indigenous population is reported to have a higher incidence of sepsis in Australia. ${ }^{8}$ Globally, case fatality rates in children vary widely with Africa having rates that are almost eight times greater, Asia 
four times greater, and South America three times greater than rates in the United States. ${ }^{5}$

Vulnerability in the Canadian healthcare context is often judged by health outcomes and the social determinants of health are often used by the Public Health Agency of Canada (PHAC) in their efforts to address the needs of vulnerable populations and to reduce health inequities as outlined in the Rio Political Declaration on Social Determinants of Health. ${ }^{9}$ Within the PHAC document, groups identified as vulnerable to health inequities are similar to those outlined in the Hennessy's manuscript report and includes indigenous groups, individuals with low income and education, rural and remote communities, and immigrants to Canada. These groups are more likely to be socioeconomically disadvantaged and are less likely to be able to access timely care. A report on frontline healthcare for vulnerable populations by the Canadian Policy Research Networks focused on groups that were deemed "unserved or underserved by the mainstream healthcare system, in particular people in rural and remote communities, people living in the inner city, and people living in conditions of poverty and low income". ${ }^{10}$ In Canada, infectious diseases in infants with Aboriginal background are several times more frequent than other regions in Canada. ${ }^{11}$ Identification of vulnerable populations in whom outcomes are poorest is the first step in the daunting challenge of designing healthcare services in Canada where the population is widely dispersed. Vulnerability may be due to geographic and other barriers to meaningful consultation to determine needs including access to preventative care and early diagnosis and treatment.

It is challenging to reconcile the lower rates of sepsis reported in this Canadian study compared with those reported in similarly developed countries. Sepsis incidence in Sweden, gleaned from a registry of hospitalized patients, ${ }^{12}$ was eight-fold higher with 780 cases per 100,000 population based on the Sepsis 3 definitions; similarly, sepsis incidence in South Korea was five times higher than in Canada. ${ }^{13}$ While geographic differences in burden and outcomes may be due to the type of infectious pathogens, genetic susceptibilities, and socio-demographic realities, the differences may also be partly explained by mechanisms used for data collection and coding. The incidence of sepsis is known to be under-estimated in hospital discharge data ${ }^{14}$ and even in the United States up to $12 \%$ of sepsis deaths occur outside of the hospital ${ }^{15}$ and hence are not captured by studies relying only on hospital discharge and electronic health records. In addition, children in Canada were not included in Hennessy's study. Children are also vulnerable to sepsis, as reported from the CIHI database over a similar timeframe. Thus, the overall burden of sepsis in Canada cannot be inferred from the Hennessy report. ${ }^{16}$ Nevertheless, this factor is unlikely to explain the lower incidence compared with that reported in adults in Sweden and South Korea.

Much remains to be done to fully unravel the burden of sepsis and health vulnerabilities in Canada. We should start by developing a robust national data set capturing salient variables that may indicate vulnerabilities and outcomes at a more granular level. This should include variables from both census and DAD data sets. Only then can we provide a sound rationale for investment of healthcare dollars to fulfill the mandate of the World Health Organization and United Nations Resolution to recognize sepsis as a global health priority and respond by improving its prevention, diagnosis, and management. ${ }^{17}$

\section{Une exploration des vulnérabilités au sepsis au Canada}

L'épidémiologie et les systèmes de surveillance du sepsis sont rudimentaires, ce qui met en évidence notre manque de connaissances concernant cette maladie aussi répandue que dévastatrice. ${ }^{1,2}$ À l'ère de coûts de santé toujours plus élevés et de ressources financières toujours plus restreintes, nous nous devons de prendre des décisions stratégiques quant à l'allocation de nos fonds en santé publique. Cette allocation devrait se fonder sur des données détaillées solides tout en tenant compte des autres priorités qui, en fonction du contexte local, rivalisent pour ces fonds. Cependant, l'acquisition et l'interprétation des données permettant de bien comprendre l'épidémiologie du sepsis ont été freinées par les signes et symptômes protéiformes $\mathrm{du}$ sepsis, une situation qui se reflète dans les fluctuations des critères diagnostiques. En raison de ces obstacles, le but de quantifier le fardeau que représente véritablement le sepsis est demeuré hors de portée.

L'identification des populations vulnérables constitue la clef de voûte de l'allocation des ressources pour la prévention du sepsis et de la fourniture de soins aigus et d'un suivi optimaux des patients qui en sont atteints. En effet, les facteurs sociodémographiques, notamment les iniquités sociales, ont traditionnellement contribué aux causes, à la dissémination et à l'évolution des maladies infectieuses et du sepsis dans le monde. ${ }^{3}$ Les archives hospitalières ne fournissent que peu d'informations quant aux facteurs sociodémographiques sous-tendant une vulnérabilité et potentiellement associés au sepsis. En raison de ce manque de données, la plupart des études rapportant les pronostics de soins critiques et hospitaliers ne sont que de peu d'utilité pour orienter l'utilisation des 
ressources, réduire les inégalités en matière de soins, ou cibler certaines stratégies de prévention du sepsis.

Dans l'optique de dresser un portrait exhaustif des caractéristiques sociodémographiques possiblement associées au sepsis, Hennessy et coll. ${ }^{4}$ ont lié les données de recensement de Statistique Canada à la Base de données sur les congés des patients (DAD, pour Discharge Abstract Database) de l'Institut canadien d'information sur la santé (ICIS). Les données du recensement canadien comportent des informations concernant le revenu, l'éducation, l'emploi, l'ethnicité, le statut de minorité visible et d'immigrant ainsi que les limitations fonctionnelles, alors que la DAD contient des données démographiques (bien que peu nombreuses), administratives et cliniques de partout au Canada (à l'exception du Québec). Les données de la DAD des patients âgés de 18 ans ou plus et admis entre 2006/2007 et 2008/2009 ont été associées aux données du recensement de 2006. Les auteurs ont découvert que le taux standardisé pour l'âge d'hospitalisation due au sepsis était de 96 cas par 100 000 personnes; parmi ces cas, 37 cas par 100000 personnes étaient catégorisés comme sepsis grave. Le couplage de ces bases de données a révélé plusieurs facteurs associés à une probabilité plus élevée d'hospitalisation pour sepsis, notamment le sexe masculin, le fait de n'avoir jamais été marié, un statut de minorité visible, les limitations fonctionnelles et le fait de ne pas faire partie de la population active. Ces variables sont très probablement le reflet d'iniquités sociodémographiques et de populations vulnérables.

Bien que les résultats de ce rapport ne soient par surprenants, la force de cette étude réside dans l'incorporation d'indices sociodémographiques qui pourraient indiquer une vulnérabilité au sepsis et à l'hospitalisation. Le fait que vulnérabilité au sepsis et iniquités soient liées se reflète dans des différences flagrantes d'incidence et d'évolution du sepsis entre les pays et à l'intérieur même des frontières nationales. ${ }^{1,2,5}$ Des rapports nous provenant d'Australie ${ }^{6}$ et des ÉtatsUnis $^{7}$ soulignent que l'iniquité en matière de ressources socioéconomiques et d'accès aux soins de santé contribue au fardeau du sepsis et à son évolution. Qui plus est, les populations autochtones sont celles présentant la plus forte incidence de sepsis en Australie. ${ }^{8}$ Globalement, les taux de létalité chez l'enfant sont très variables, l'Afrique affichant des taux presque huit fois plus élevés, l'Asie quatre fois plus élevés, et l'Amérique du Sud trois fois plus élevés que les taux rapportés aux États-Unis. ${ }^{5}$

La vulnérabilité, dans le contexte des soins de santé canadiens, est souvent évaluée en fonction des devenirs de santé et les déterminants sociaux de santé sont fréquemment utilisés par l'Agence de la santé publique du Canada (ASPC) dans ses efforts pour répondre aux besoins des populations vulnérables et réduire les iniquités en matière de santé, tel qu'énoncé dans la Déclaration politique de Rio sur les déterminants sociaux de la santé. ${ }^{9}$ Dans le document de l'ASPC, les groupes identifiés comme étant vulnérables aux iniquités en matière de santé recoupent ceux décrits dans le rapport de Hennessy et comprennent les groupes indigènes, les personnes à faible revenu et n'ayant que peu de scolarité, les collectivités rurales et éloignées, et les immigrants au Canada. Ces groupes courent un risque plus élevé d'être désavantagés d'un point de vue socioéconomique et de ne pas avoir accès à des soins en temps opportun. Un rapport portant sur les soins de santé de première ligne destinés aux populations vulnérables par les Réseaux canadiens de recherche en politiques publiques s'est intéressé aux groupes jugés «non desservis ou sous-desservis par le système de soins de santé officiel, et en particulier aux personnes dans les collectivités rurales ou éloignées, aux personnes vivant dans les quartiers défavorisés et aux personnes vivant dans des conditions de pauvreté et de faible revenu $»{ }^{10} \mathrm{Au}$ Canada, les maladies infectieuses contractées par les nourrissons d'origine autochtone sont beaucoup plus fréquentes que dans les autres régions du pays. ${ }^{11}$ L'identification des populations vulnérables chez lesquelles les pronostics sont les moins bons constitue la première étape du défi de taille que représente la conception de services de santé au Canada, un pays où la population est très dispersée. Cette vulnérabilité pourrait être causée, entre autres, par des obstacles géographiques à une consultation significative afin de déterminer les besoins à combler, y compris un accès à des soins de prévention, à un diagnostic opportun et à un traitement.

Il est difficile de réconcilier les faibles taux de sepsis rapportés dans cette étude canadienne à ceux rapportés dans des pays tout aussi développés. En Suède par exemple, l'incidence de sepsis telle que recueillie à partir d'un registre de patients hospitalisés ${ }^{12}$ était huit fois plus élevée, avec 780 cas par 100000 habitants selon les définitions du Sepsis 3; de la même façon, l'incidence de sepsis en Corée du Sud était cinq fois plus élevée qu'au Canada. ${ }^{13}$ Bien que les différences géographiques en matière de fardeau et de pronostic puissent être dues au type de pathogènes infectieux, aux susceptibilités génétiques ainsi qu'aux réalités sociodémographiques, les différences pourraient également partiellement s'expliquer par les mécanismes utilisés pour la collecte et l'encodage des données. On sait que l'incidence de sepsis est sousestimée dans les données de congé d'hôpital ${ }^{14}$ et, même aux États-Unis, jusqu'à $12 \%$ des décès dus au sepsis surviennent hors du milieu hospitalier ${ }^{15}$; ainsi, ces décès ne sont pas comptabilisés dans les études s'appuyant exclusivement sur les données de congé d'hôpital et les dossiers de santé informatisés. En outre, les enfants n'ont 
pas été inclus dans l'étude canadienne de Hennessy. Les enfants sont également vulnérables au sepsis, comme le rapporte la base de données de l'ICIS au cours d'une période semblable. Ainsi, on ne peut déduire quel est le véritable fardeau global du sepsis au Canada à partir du rapport de Hennessy. ${ }^{16}$ Toutefois, ce facteur seul n'explique probablement pas l'incidence plus faible de sepsis au pays comparativement à celle rapportée chez les adultes en Suède et en Corée du Sud.

Il reste encore beaucoup à faire avant de pouvoir décrire pleinement le fardeau du sepsis et des vulnérabilités en santé au Canada. Nous devrions commencer par la mise au point d'un ensemble de données national robuste qui capturerait les variables saillantes capables d'indiquer les vulnérabilités et les pronostics de façon plus détaillée. Cela devrait inclure des variables tirées tant des ensembles de données du recensement que de celles de la DAD. Alors seulement pourrons-nous fournir des justifications solides pour investir nos dollars destinés aux soins de santé afin de remplir le mandat de la Résolution de l'Organisation mondiale de la Santé et des Nations Unies visant à reconnaître l'état septique comme une priorité de santé mondiale et d'y répondre en améliorant sa prévention, son diagnostic et sa prise en charge. ${ }^{17}$

\section{Conflicts of interest None.}

Funding statement None.

Editorial responsibility This submission was handled by Dr. Sangeeta Mehta, Associate Editor, Canadian Journal of Anesthesia.

\section{Conflit d'intérêt Aucun.}

\section{Déclaration de financement Aucune.}

Responsabilité éditoriale Cet article a été traité par Dre Sangeeta Mehta, rédactrice adjointe, Journal canadien d'anesthésie.

\section{References}

1. Fleischmann C, Scherag A, Adhikari NK, et al. Assessment of global incidence and mortality of hospital-treated sepsis. Current estimates and limitations. Am J Respir Crit Care Med 2016; 193 : 259-72.

2. Fleischmann-Struzek C, Goldfarb DM, Schlatttmann $P$, Schlapbach LJ, Reinhart K, Kissoon N. The global burden of paediatric and neonatal sepsis: a systematic review. Lancet Respir Med 2018; 6: 223-30.

3. Farmer $P$. Social inequalities and emerging infectious diseases. Emerg Infect Dis 1996; 4: 259-69.
4. Hennessy D, Soo A, Niven DJ, et al. Socio-demographic characteristics associated with hospitalization for sepsis in Canada: a census linked cohort study. Can J Anesth 2020; 67: https://doi.org/10.1007/s12630-019-01536-z.

5. Tan B, Wong JJ, Sultana $R$, et al. Global case-fatality rates in pediatric severe sepsis and septic shock: a systematic review and meta-analysis. JAMA Pediatr 2019; 173: 352-62. Correction: JAMA Pediatr 2019; 173: 401.

6. Evidence Or T, Advisor Evaluation. Trends and disparities in sepsis hospitalisations in Victoria. Australia. Australian Health Rev 2016; 40: 511-8.

7. Wang HE, Devereaux RS, Yealy DM, Safford MM, Howard G. National variation in United States sepsis mortality: a descriptive study. Int J Health Geogr 2010; . https://doi.org/10.1186/1476072X-9-9.

8. Davis JS, Cheng AC, McMillan M, Humphrey AB, Stephens DP, Anstey NM. Sepsis in the tropical top end of Australia's Northern Territory: disease burden and impact on Indigenous Australians. Med J Aust 2011; 194: 519-24.

9. Public Health Agency of Canada. Rio political declaration on social determinants of health: a snapshot of Canadian actions 2015. CHer Majesty the Queen in Right of Canada, as represented by the Minister of Health, 2015. Available from URL: http://www.healthycanadians.gc.ca/publications/scienceresearch-sciences-recherches/rio/alt/rio2015-eng.pdf (accessed October 2019)

10. Hay D, Varga-Toth J, Hines E. Frontline Health Care in Canada: Innovations in Delivering Services to Vulnerable Populations. Research Report Fl63 Family Network. September 2006. Available from URL: http://ircp.info/Portals/11/Downloads/ Policy/CPRN\%20Frontline\%20Healthcare\%20in\%20Canda.pdf (accessed October 2019).

11. Collins SA, Sumala P, Osborne G, et al. Causes and risk factors for infant mortality in Nunavut, Canada 1999-2011. BMC Pediatr 2012; . https://doi.org/10.1186/1471-2431-12-190.

12. Mellhammar L, Wullt S, Lindberg A, Lanbeck $P$, Christensson B, Linder A. Sepsis incidence: a population-based study. Open Forum Infect Dis 2016; . https://doi.org/10.1093/ofid/ofw207.

13. Kim J, Kim K, Lee H, Ahn S. Epidemiology of sepsis in Korea: a population-based study of incidence, mortality, cost and risk factors for death in sepsis. Clin Exp Emerg Med 2019; 6: 49-63.

14. Fleischmann-Struzek C, Thomas-Ruddel DO, Schettler A, et al. Comparing the validity of different ICD coding abstraction strategies for sepsis case identification in German claims data. PLoS One 2018; . https://doi.org/10.1371/journal.pone.0198847.

15. Melamed A, Sorvillo FJ. The burden of sepsis-associated mortality in the United States from 1999 to 2005: an analysis of multiple-cause-of-death data. Crit Care 2009; https://doi.org/ $10.1186 / \mathrm{cc} 7733$.

16. Thompson GC, Kissoon N. Sepsis in Canadian children: a national analysis using administrative data. Clin Epidemiol 2014; 6: 461-9.

17. Reinhart K, Daniels R, Kissoon N, Machado FR, Schachter RD, Finfer $S$. Recognizing sepsis as a global health priority - a WHO resolution. N Engl J Med 2017; 377: 414-7.

Publisher's Note Springer Nature remains neutral with regard to jurisdictional claims in published maps and institutional affiliations. 